Article

\title{
Strategy to Explore Magnetized Cosmic Web with Forthcoming Large Surveys of Rotation Measure
}

\author{
Takuya Akahori (D) \\ Mizusawa VLBI Observatory, National Astronomical Observatory of Japan (NAOJ), 2-21-1 Osawa, Mitaka, \\ Tokyo 181-8588, Japan; takuya.akahori@nao.ac.jp
}

Received: 3 September 2018; Accepted: 13 November 2018; Published: 16 November 2018

\begin{abstract}
The warm-hot intergalactic medium (WHIM) is a candidate for the missing baryons in the Universe. If the WHIM is permeated with the intergalactic magnetic field (IGMF), the Faraday rotation measure (RM) of the WHIM is imprinted in linearly-polarized emission from extragalactic objects. In this article, we discuss strategies to explore the WHIM's RM from forthcoming radio broadband and wide-field polarization sky surveys. There will be two observational breakthroughs in the coming decades; the RM grid and Faraday tomography. They will allow us to find ideal RM sources for the study of the IGMF and give us unique information of the WHIM along the line of sight.
\end{abstract}

Keywords: intergalactic medium; large-scale structure of the Universe; magnetic fields; polarization; techniques; polarimetric

\section{Introduction}

\subsection{Magnetized Cosmic Web}

The standard theory of big-bang nucleosynthesis predicts the total amount of baryons in the Universe. Since the importance of studying the baryon budget was pointed out [1,2], cosmic baryons are being explored and found as galaxies, the intergalactic medium (IGM) in galaxy clusters, quasar absorption-line systems, etc. However, confirmed baryons in the local Universe comprise only about $60 \%$, and the rest are still missing [3]. This is a longstanding problem of the standard cosmology. Cosmological structure-formation simulations suggest that the majority of the missing baryons exist as the warm-hot intergalactic medium (WHIM), which resides mostly in galaxy filaments with temperature from $10^{5} \mathrm{~K}-10^{7} \mathrm{~K}$. Exploring the WHIM in galaxy filaments is a straightforward way to solve this problem, although no firm observational detection of the WHIM in galaxy filaments has been made.

The IGM, including the WHIM, is most likely magnetized. There are diverse mechanisms of magneticfield generation, amplification and maintenance in different epochs [4,5]. For example, the intergalactic magnetic field (IGMF) is generated in the recombination [6], in the first-star formation [7,8], in the reionization $[9,10]$ and in the structure formation [11] of the Universe. Seed magnetic fields can be amplified through a compression and turbulence dynamo in the structure formation; see, e.g., [12] and the references therein. Leakages of galactic cosmic rays and magnetic fields by jets, winds and ram pressure stripping are another source of the IGMF [13-15]. A range for the WHIM's IGMF strength seems to be $O(1-100)$ nG.

The IGMF plays essential roles in various fields of astrophysics [16]. The IGMF imprints its existence in the spectrum of the Cosmic Microwave Background [17], deflects propagations of ultra-high energy cosmic rays [18] and produces blazar's $\gamma$-ray halo [19]. The IGMF is important 
to understand thermal balance in cool-core clusters [20] and non-thermal energy in merging clusters [21,22]. Moreover, the IGMF influences the configuration of magnetic fields in galaxies [23]. These examples tell us the fact that understanding the IGMF is of crucial importance. While it is still challenging to handle the IGMF in cosmological simulations [4,12], detection and observational diagnostics of the IGMF in galaxy filaments are extremely important. A firm detection of the IGMF in galaxy filaments brings the discovery of the missing baryons, as well.

\subsection{Faraday Rotation Measure}

Observing Faraday rotation of linear polarization is one of only a few possible ways to measure the extragalactic magnetic field. This article highlights rotation measure (RM) to be studied at a centimeter wavelength. Another possible way, synchrotron radiation from cosmological shock waves, is out of scope; see, e.g., [24,25]; some articles in this volume.

Faraday rotation is the rotation of the polarization angle due to the birefringence of the magneto-ionic medium. RM from a source located at the redshift, $z_{\mathrm{s}}$, to an observer at $z=0$ is given by:

$$
R M\left(z_{\mathrm{s}}\right)=C_{\mathrm{R}} \int_{z_{\mathrm{s}}}^{0} \frac{n_{\mathrm{e}}(z) B_{\|}(z)}{(1+z)^{2}} \frac{d l(z)}{d z} d z \operatorname{rad~m}^{-2},
$$

where $n_{\mathrm{e}}(z)$ is the electron density at the redshift $z$ in units of $\mathrm{cm}^{-3}, B_{\|}(z)$ the line-of-sight (LOS) magnetic-field strength at $z$ in $\mu \mathrm{G}, d l(z)$ the LOS line element at $z$ in kpc and $C_{R} \simeq 811.9$ the constant. An extragalactic linearly-polarized fast radio burst (FRB) also gives the dispersion measure (DM),

$$
\operatorname{DM}\left(z_{\mathrm{s}}\right)=C_{\mathrm{D}} \int_{0}^{z_{\mathrm{s}}} \frac{n_{\mathrm{e}}(z)}{(1+z)} \frac{d l(z)}{d z} d z \mathrm{pc} \mathrm{cm}^{-3},
$$

with $C_{\mathrm{D}} \simeq 1000$. The mean LOS strength of WHIM's IGMF can be estimated by:

$$
B_{\|}^{\ddagger}=\frac{\langle 1+z\rangle}{f_{D M}} \frac{C_{D} R M}{C_{R} D M}
$$

where $\langle 1+z\rangle \sim 1.0-1.5$ and $f_{D M} \sim 0.5$ are the correction factors, both of which can be evaluated for a given cosmology model solely from the DM; see [26]. $B_{\|}^{\ddagger}$ is the density-weighted average and is about one order of magnitude larger than the volume average in the case of the WHIM [27].

RM study was notably developed in the last decade [28,29], and it will be advanced more dramatically in the Square Kilometre Array (SKA) era [30]. There are many RM observations for galaxy clusters [31]. $\mathrm{RM}$ is $O(100) \mathrm{rad} \mathrm{m}^{-2}$ through the central regions of normal clusters, or 10-100-times larger in cool-core clusters, and decreases to several tens of $\mathrm{rad} \mathrm{m}^{-2}$ toward outskirts. The field strength around the cluster center is estimated to be $O(1-10) \mu \mathrm{G}$ with the maximum scale of $O(100) \mathrm{kpc}$, assuming the Kolmogorov-like turbulence and the energy equipartition between cosmic rays and the magnetic field; see, e.g., [32]. The fact that a hotter/X-ray-brighter system has a larger variance of RM [33] may imply a relation with the development of magneto-hydrodynamic turbulence, though the real origin and history of the IGMF are a longstanding mystery [34,35].

\subsection{Prediction of the WHIM's RM}

There is so far no firm detection of WHIM's RM. Figure 1 shows a theoretical prediction of WHIM's RM [36,37]. This is made with data of structure-formation simulations adopting a physically-motivated IGMF model [27]. The LOS profiles (the left panel) show that the sign of RM changes as the direction of the IGMF changes. Consequently, an integration of WHIM's RM along the LOS behaves like a random walk process with the coherence length of the IGMF several hundred kpc, smaller than the depth of a filament. The integrated WHIM's RM mostly comes from the RM at the density peak of the filament, meaning that the integrated WHIM's RM is sensitive to dense parts of filaments. 
The middle panels of Figure 1 show RM maps through the cosmic web up to the redshifts of 0.1 and 5.0. In each image pixel, RM is integrated from a source following the probability distribution function of redshift for radio galaxies [38], to the observer located at the center of a local group; see [36] for details. In the local Universe, galaxy clusters and groups have RM $O(100) \mathrm{rad} \mathrm{m}^{-2}$ and $O(10)$

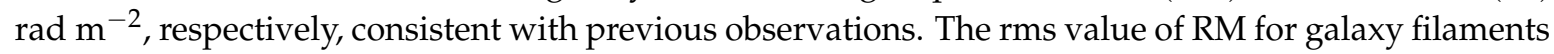
(WHIM) is $1.4 \mathrm{rad} \mathrm{m}^{-2}$, which is in agreement with the turbulent dynamo scenario [39].

Integrated toward high redshift, the sky is filled with the cosmic RM background by WHIM's RM. There is a spatial correlation in the integrated RM map; the second-order structure function (SF) of the RM map is flat down to $\sim 0^{\circ} .1$ with $O(100) \mathrm{rad}^{2} \mathrm{~m}^{-4}$ and then decreases to $O(10) \mathrm{rad}^{2} \mathrm{~m}^{-4}$ at $\sim 0^{\circ} .01$ [40]. A gradient of WHIM's RM is negligibly small in a beam size of modern interferometers around $1 \mathrm{GHz}$, say $1^{\prime \prime}-1^{\prime}$. Therefore, beam depolarization by WHIM's RM is minor. The right panel of Figure 1 shows the rms values of the RM maps, where RM is integrated up to the redshift of 5.0. The rms values increase with redshift and then saturate around $10 \mathrm{rad} \mathrm{m}^{-2}$, except the case including galaxy clusters. The saturation is caused by the decrease of RM by $(1+z)^{-2}$ in Equation (1), the decrease of the number of sources and the decrease of the proper distance at high redshift.
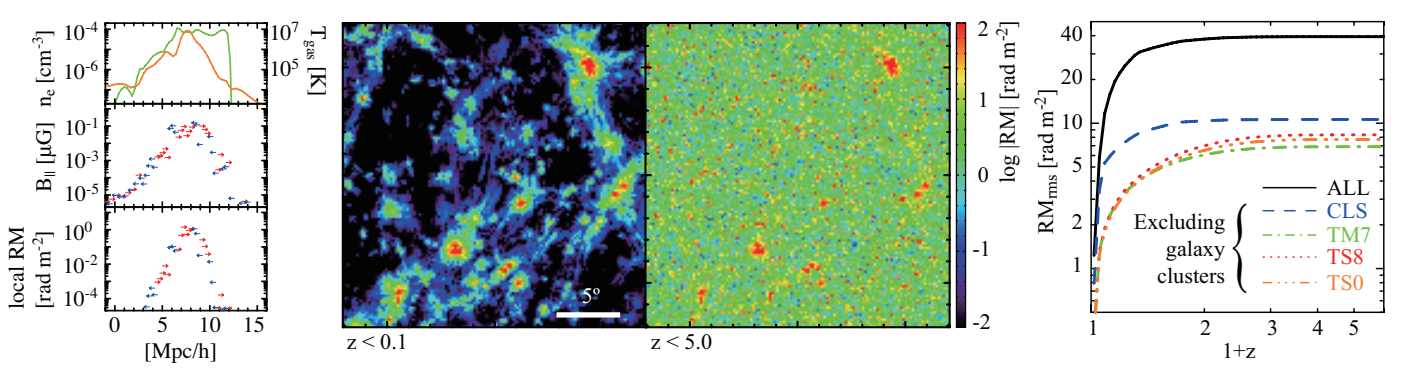

Figure 1. (Left) One-dimensional profiles of the electron density $n_{\mathrm{e}}$ (green), the gas temperature $T_{\text {gas }}$ (orange), the line-of-sight component of the intergalactic magnetic field (IGMF) $B_{||}$and the local rotation measure (RM) for a $195-\mathrm{h}^{-1} \mathrm{kpc}$ spatial resolution, through a typical filament in the local Universe. Here, $h$ is the Hubble parameter. The arrows indicate the signs of $B_{\|}$and the local RM [37]. (Middle) RM maps of $200 \mathrm{deg}^{2}$ considered up to $z<0.1$ (the local Universe, left) and $z<5$ (right). (Right) Redshift evolution of the rms value of RM. The average for 200 (runs) $\times 2048^{2}$ (pixels in $200 \mathrm{deg}^{2}$ ) is shown. Except ALL, galaxy clusters were excluded from the integrations using different criteria (CLS, TM7, TS8and TS0) [36].

\subsection{Observational Issues}

Cosmic variance of the IGMF is an issue in discussing the origin of the IGMF. However, it was recently suggested that a large sample of FRBs will likely be able to discriminate between extreme scenarios for the origin of the IGMF [41]. According to the previous works [36,42], a field of view of a few tens of square degrees is required to overcome the variance and to obtain universal results for the WHIM's RM.

Contaminations from other RMs along the LOS are another problem. Recently, a pioneering work was achieved with FRB150807 [43]. They argued LOS components in the observed RM and derived an upper limit of the IGMF strength, $<21 \mathrm{nG}$, which does not contradict theoretical predictions. Further understanding of the contaminations is key for a firm detection of the WHIM's RM. In the near future, we could overcome the contamination problem by the two methods, the RM grid and Faraday tomography, introduced in the rest of this article.

\section{The Power of RM Grid}

Spatially-compact, linearly-polarized sources such as pulsars, quasars and FRBs can be utilized as data points of RM on the sky. These data points are called the RM grid in the context of SKA cosmic magnetism [44]. A representative science case using the RM grid is the all-sky RM map [45,46], which 
reveals the global magnetic-field structure of the Milky Way. Another important science case of the RM grid is the statistical study of LOS components of RM. The gray dots in Figure 2 show the observed RMs for 317 extragalactic sources at high galactic latitudes, where RM and redshift were taken from the previous works $[45,47]$. The black line shows the standard deviation of RMs, binned with redshift ranges, $z=0-0.2$ (71 sources), 0.2-0.4 (50), 0.4-0.6 (31), 0.6-1.0 (55), 1.0-2.0 (77) and 2.0-4.0 (33), where errors $\left(10 \mathrm{rad} \mathrm{m}^{-2}\right)$ are subtracted; see [42]. Although they still have large uncertainties, the observed RMs seem to be independent of redshift.

SKA Phase 1 will deliver a denser RM grid of $\sim 100 / \mathrm{deg}^{2}$ (currently $\sim 1 / \mathrm{deg}^{2}$ ) [44]. In other words, we will obtain RM samples of $\sim 4$ millions (currently $\sim 40,000$ ) in the whole sky. These data will dramatically improve the statistics and will reveal whether extragalactic RMs depend on the redshift or not. The large sample will also help us to find the RM sources, which are ideal for the study of WHIM's RM. Below, we present a simple scenario to distinguish LOS components of RM.

\subsection{Intrinsic RM and Cosmological Redshift}

Extragalactic polarized sources can possess the magneto-ionic media, which contribute to the observed RM. The average of such RMs in any redshift range should be zero, while their standard deviation, $\sigma_{\mathrm{INT}}(z)$, can be non-zero. A baseline scenario is that $\sigma_{\mathrm{INT}}(z)$ does not evolve with redshift, i.e., $\sigma_{\mathrm{INT}}(z)=\sigma_{\mathrm{INT}}(0)$. The intrinsic RM then contributes to the observed RM with a factor of $1 /(1+z)^{2}$ due to the redshift of wavelength. Therefore, the contribution can be written as $\sigma_{\text {INT }}=$ $\sigma_{\mathrm{INT}}(z)(1+z)^{-2} \sim \sigma_{\mathrm{INT}}(0)(1+z)^{-2}$, shown as the blue line in Figure 2 . Here, $\sigma_{\mathrm{INT}}(0) \sim 10 \mathrm{rad} \mathrm{m}^{-2}$ is expected [42]. This model predicts that the standard deviation of intrinsic RM for the 317 sources is $\sim 3.1 \mathrm{rad} \mathrm{m}^{-2}$, and it can be overtaken by WHIM's RM, shown as the red line.

Another scenario is that the observed (redshift-independent) RMs mostly come from intrinsic RM, i.e., intrinsic RM increases following $\sigma_{\mathrm{INT}}(z) \propto(1+z)^{2}$. Indeed, cosmological simulations suggest that a starburst protogalaxy can possess very large intrinsic RM; see Figure 14 of [48]. Such a source is not suited to the study of WHIM's RM. We expect that it exhibits a strong depolarization feature caused by a turbulent magnetic field $[49,50]$, so that we could discard it from the analysis.
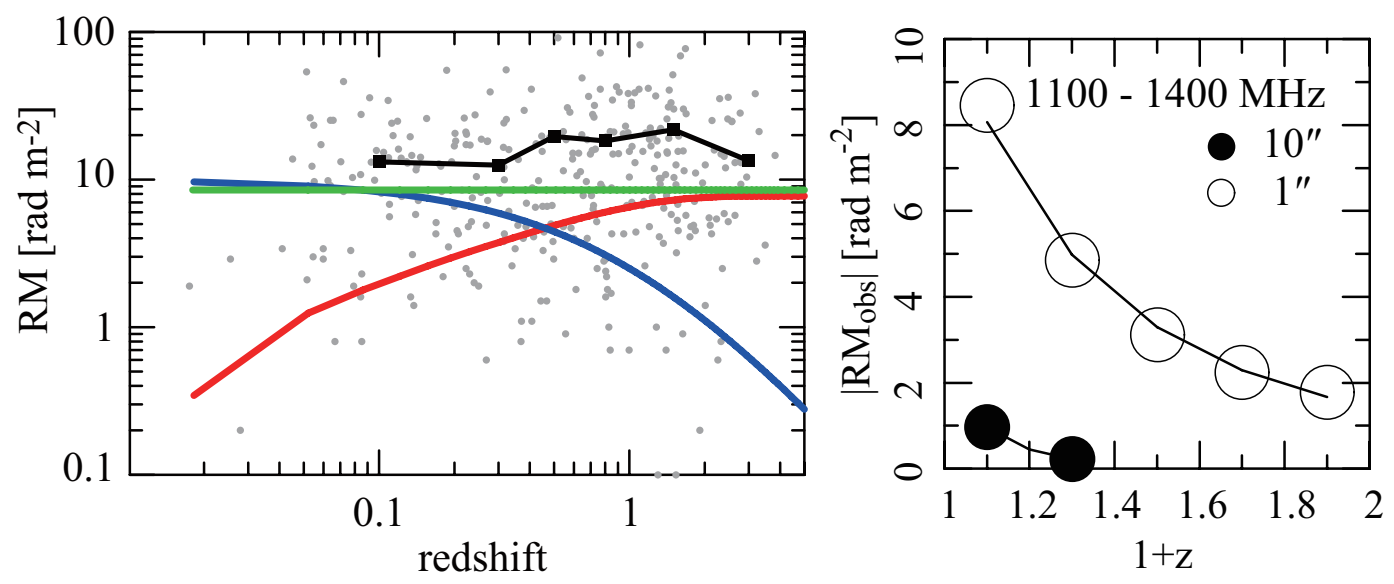

Figure 2. (Left) RM as a function of redshift [42]. Gray filled circles show the observed RMs of 317 sources located at high galactic latitudes, $|b|>75^{\circ}$. The black line indicates the standard deviation of error-subtracted RMs (see the text). The blue line shows a baseline scenario for the standard deviation of the intrinsic RM, $\sigma_{\mathrm{INT}}=10(1+z)^{-2} \mathrm{rad} \mathrm{m}^{-2}$. The red line shows the standard deviation of the WHIM's RM in the TS0 run (Figure 1 right). The green line shows the standard deviation of the observed Milky Way contribution $\sigma_{\mathrm{ISM}}=8.4 \mathrm{rad} \mathrm{m}^{-2}$ toward high galactic latitudes. (Right) Simulated RM caused by a depolarizing intervening galaxy (DING). RM is derived from the linear fit for polarization angles from $1100 \mathrm{MHz}-1400 \mathrm{MHz}$. The open and filled circles show the results with the sizes of background sources, $1^{\prime \prime}$ and $10^{\prime \prime}$, respectively. 


\subsection{Intervening RM and Faraday Depolarization}

Interstellar medium (ISM) and circumgalactic medium (CGM) of external galaxies intervening the LOS can contribute to the observed RM. We can simulate such a situation using a model of a galaxy. The right panel of Figure 2 shows an example of the simulation, where a Milky Way model with global (coherent), random (turbulent) and wind components all included and the average of 100,000 realizations of random viewing angles are shown (Akahori et al., in preparation). This suggests that the intervening RM becomes minor if it is located at relatively high redshift (say, $z>0.5$ ).

The scale of the RM structure associated with an intervening galaxy is generally smaller than an observing beam size. Therefore, this RM and polarization degree are a function of wavelength as a result of depolarization. Such a depolarization signal can be a powerful probe for identifying the depolarizing intervening galaxies (DINGs). The chance of an encounter with a DING along a LOS is roughly 0.5 ; see [51], which corresponds to the fraction of sources with the polarization degree smaller than $4 \%$ (1874 out of 3650 in [47]). It has also been argued that RM induced by the DING correlates with optical absorption-line systems [52]. Hence, DINGs can be identifiable via optical spectroscopy, as well. Zhu, \& M'enard [53] claimed 40,429 Mg II absorbers in 84,534 SDSS quasars. Again, about a half of sources will be discarded for the study of WHIM's RM.

\subsection{Galactic RM and Image Processing}

The ISM in the Milky Way Galaxy can contribute to the observed RM. An observed estimate of the standard deviation of galactic RM is $8.4 \pm 0.1 \mathrm{rad} \mathrm{m}^{-2}$ [54]. A theoretical work predicts that the standard deviation of galactic RM for a $30^{\circ} \times 30^{\circ}$ FOV toward the South Galactic Pole was $\sim 5 \mathrm{rad} \mathrm{m}^{-2}$ [40]. The RM map toward high galactic latitudes has structures of $\sim 1^{\circ}-10^{\circ}$ scales, while the power spectrum of WHIM's RM has a broad plateau over $\sim 0^{\circ} .1-1^{\circ}$ with a peak around $\sim 0^{\circ} 15$ (Figure 3, left). Therefore, the galactic large-scale RM component can be reasonably filtered out by high-pass filter image processing in the Fourier domain. Practically, this is achieved by subtracting a smoothed map from the observed one and obtaining a residual RM map, where the smoothing length should be a few degrees [42].

The right panel of Figure 3 shows the SFs toward the South Galactic Pole. The simulated SF of galactic RM has difficulty explaining both the amplitude and the flat slope of the observed SFs. The comparison between simulated and observed SFs implies that a substantial fraction of the RM toward high galactic latitudes may be attributed to the IGMF, though the errors at small-angular scales in the observations are still too large to allow any concrete conclusion.
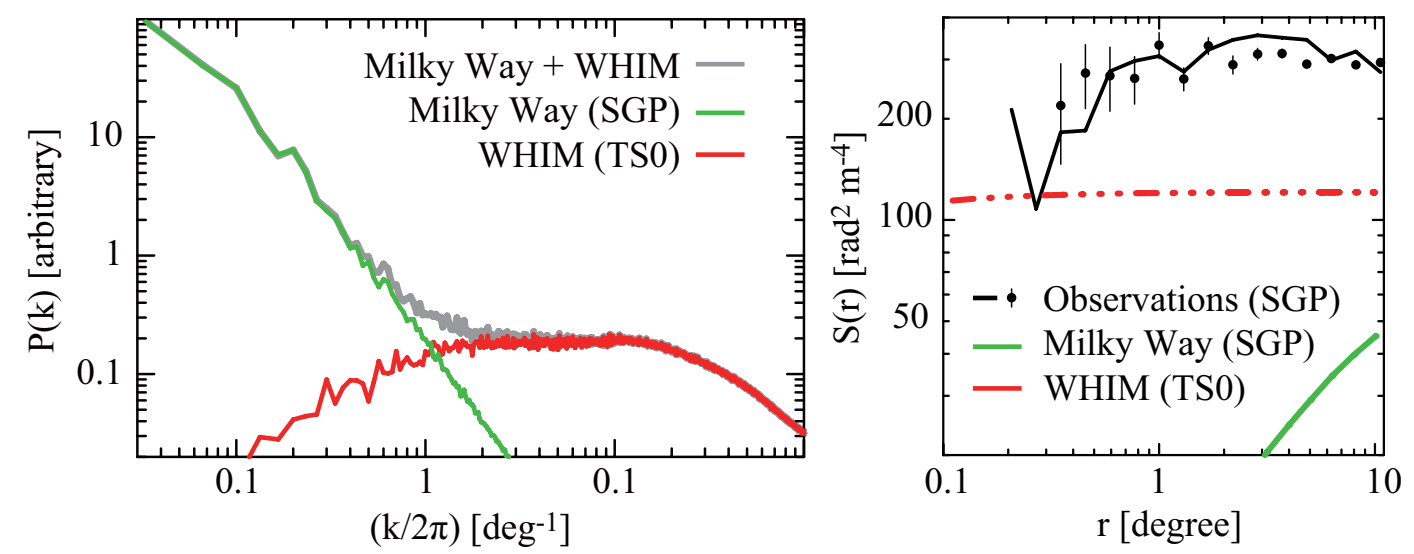

Figure 3. (Left) Simulated power spectra of RM for the ISM toward the South Galactic Pole (blue), the warm-hot intergalactic medium (WHIM) (red) and their sum (gray) [42]. (Right) The second-order structure functions of RM toward the South Galactic Pole [36,40]. Black marks [55] and line [56] are observational results. 


\subsection{Atmospheric RM and Calibration}

Finally, instrumental noise and calibration error are uncertainties in RM measurement. Ionospheric and tropospheric contributions are significant at low and high frequencies, respectively. Since our target $\mathrm{RM}$ is several to ten $\mathrm{rad} \mathrm{m}^{-2}$, a sweet spot of frequency coverage is expected to be the UHF band (300-3000 MHz; see the next section), where the former contribution is more important.

The errors in existing data around GHz are still large; $8 \mathrm{rad} \mathrm{m}^{-2}$ for VLA RMs [56] and 3-5 $\mathrm{rad} \mathrm{m}^{-2}$ for ATCA and WSRT data [55]. The errors will be decreased to a few $\mathrm{rad} \mathrm{m}^{-2}$ for the SKA MID Band 2, because the sensitivity and polarization purity of the instrument will be improved and atmospheric variation will be well-monitored and calibrated. Observations at low frequency such as LOFAR and MWA give a fine RM resolution with a low absolute noise. For instance, Sotomayor-Beltran et al. [57] reported calibration of ionospheric RMs with absolute errors of $\sim 0.1 \mathrm{rad} \mathrm{m}^{-2}$.

\section{The Power of Faraday Tomography}

Faraday tomography or Faraday RM synthesis $[58,59]$ is thought to be a revolutionary technique in cosmic magnetism. In this section, we discuss the power of Faraday tomography for the study of WHIM's RM. The following works do not address the technical problems of Faraday tomography. Improvement of Faraday tomography such as RM CLEAN and QU-fitting [60-64] is another important work; see also some articles in this volume.

\subsection{Find the FDF Gap Caused by the WHIM}

Faraday tomography reconstructs the Faraday dispersion function (FDF) or the Faraday spectrum (Figure 4 left), which is obtained from Fourier transformation of the complex polarized-intensity spectrum (Figure 4 right). The FDF could unveil multiple RM components along the LOS and would be useful for studying WHIM's RM. It is important to understand what the WHIM's RM looks like in the FDF, because without this understanding, we cannot recognize the signal of WHIM's RM.

Two cases of observations were studied previously [51], (i) the background compact source behind the diffuse foreground source and (ii) two pair compact sources. Figure 4 shows an example of Case (i). We assume to be able to find a spatially-compact, Faraday-thin (its FDF looks like a delta function) extragalactic source such as quasars, radio galaxies and FRBs. We also suppose that there is no DING toward the source. This source then appears at a certain Faraday depth $(\phi)$ induced by RMs due mostly to the IGMF and the Milky Way. The Milky Way can be bright and likely appears as a Faraday-thick source, so that the gap between the two signals in $\phi$ space corresponds to RM due to the IGMF if the signs of RMs for the Milky Way and the WHIM are the same [51].
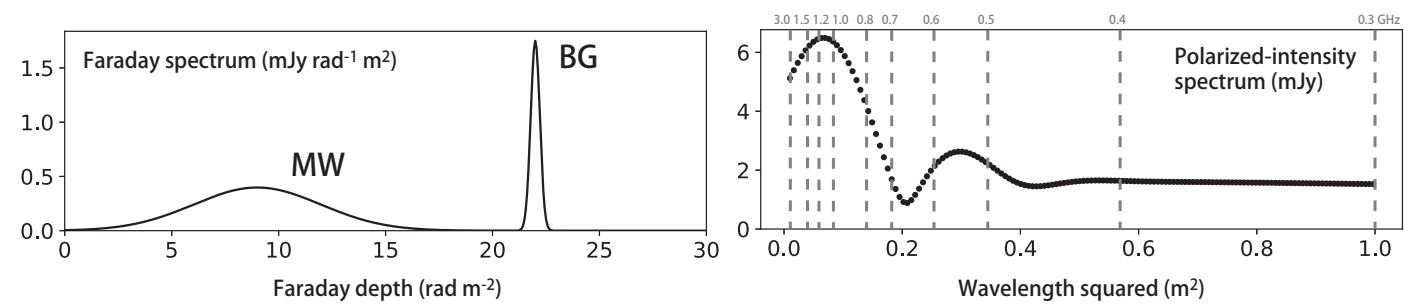

Figure 4. (Left) Faraday spectrum. (Right) Polarized intensity spectrum.

The above situation is modeled with two Gaussian functions as follows:

$$
F(\phi)=\sum_{i=\mathrm{MW}, \mathrm{BG}} \frac{f_{i} e^{2 i \theta_{i}}}{\sqrt{2 \pi} \delta \phi_{i}} \exp \left\{-\frac{\left(\phi-\phi_{i}^{\prime}\right)^{2}}{2 \delta \phi_{i}^{2}}\right\},
$$


where the subscripts MW and BG represent a Milky Way foreground and an extragalactic background, respectively. RM due to the IGMF is defined by truncating the Gaussian tail at three times the standard deviation:

$$
R M_{\mathrm{IGMF}}=\left(\phi_{\mathrm{BG}}^{\prime}-3 \delta \phi_{\mathrm{BG}}\right)-\left(\phi_{\mathrm{MW}}^{\prime}+3 \delta \phi_{\mathrm{MW}}\right) .
$$

The Faraday depth of the Milky Way depends on galactic longitude and latitude. It is $\sim+6 \mathrm{rad} \mathrm{m}^{-2}$ toward the South Galactic Pole $[40,46]$, and its absolute value is larger at lower galactic latitudes. We will fit an observed FDF with this model and estimate WHIM's RM.

\subsection{Optimum Frequency for Exploring the IGMF}

It is important to understand which frequency is essential for exploring WHIM's RM, because the performance of Faraday tomography is primarily determined by frequency covering of the data. Even though SKA will achieve broadband observation, seamless and complete frequency coverage is difficult because the data at some frequencies are not available due to artifacts such as radio frequency interferences and receiver band edges/gaps. Therefore, we will explore the FDF gap from an incomplete FDF, which is reconstructed from the limited data.

We can investigate optimum frequency coverage using a simple FDF model described above [65]. We estimate confidence intervals of the model parameters by means of the Fisher information matrix [66]. Given the data at 1400-1420 MHz and 1500-1600 MHz, let us explore the optimum frequency of the $\mathrm{P}_{*}$ band, which has a $20-\mathrm{MHz}$ or $40-\mathrm{MHz}$ bandwidth and is in $300-1000 \mathrm{MHz}$. Figure 5 shows the error profiles between the input $R M_{\mathrm{IGMF}}$ and the center frequency of the $\mathrm{P}_{*}$ band. We find that our choice of multiple narrowband data in the UHF provides a reasonable constraint on WHIM's RM. For example, toward high galactic latitudes $\left(\delta \phi_{\mathrm{MW}}=2 \mathrm{rad} \mathrm{m}^{-2}\right.$; left panel of Figure 5), $\mathrm{RM}_{\mathrm{IGMF}} \sim 10 \mathrm{rad} \mathrm{m}{ }^{-2}$ is detectable with less than $10 \%$ error, if we choose the $\mathrm{P}_{*}$-band center around 400-700 MHz with a 40-MHz bandwidth. The optimum frequency depends on the Faraday thickness $\left(\delta \phi_{\mathrm{MW}}\right)$ of the Milky Way foreground, as shown in Figure 5. Our simulations also suggest that exceptionally bright sources such as FRBs are applicable for this work; see [65].
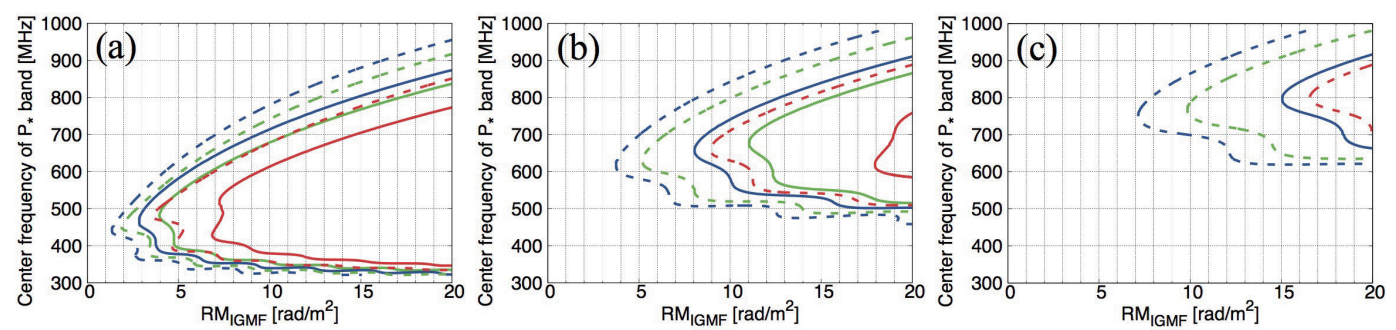

Figure 5. Error profiles between the input $R M_{\mathrm{IGMF}}$ and the $\mathrm{P}_{*}$-band center. Results for $\delta \phi_{\mathrm{MW}}=2$, 4, $6 \mathrm{rad} \mathrm{m}^{-2}$ are shown from $(\mathbf{a}-\mathbf{c})$, respectively. The blue, green and red lines show the contours on which $R M_{\text {IGMF }}$ is determined with statistical errors of $30 \%, 20 \%$ and $10 \%$, respectively. The solid and dashed lines are the results with $20-\mathrm{MHz}$ and $40-\mathrm{MHz}$ bandwidths of the $\mathrm{P}_{*}$ band, respectively.

\section{Concluding Remarks}

This article summarized theoretical predictions of WHIM's RM and discussed observational strategies to confront issues on measuring WHIM's RM. Forthcoming radio broadband and wide-field polarization sky surveys bring two technical breakthroughs for cosmic magnetism, the RM grid and Faraday tomography, both of which allow us to find ideal RM sources to detect WHIM's RM. The SKA science community is planning all-sky surveys at Band 2 [44]. Our result suggests that a Band 1 follow-up is very important for the study of WHIM's RM by means of Faraday tomography.

Funding: This work was supported in part by JSPS KAKENHI Grant Number JP17K01110. Numerical computations were carried out on the PC cluster at the Center for Computational Astrophysics, National Astronomical Observatory of Japan. 
Conflicts of Interest: The author declares no conflict of interest.

\section{References}

1. Cen, R.; Ostriker, J.P. Where Are the Baryons? Astrophys. J. 1999, 514, 1-6. [CrossRef]

2. Fukugita, M.; Hogan, C.J.; Peebles, P.J.E. The Cosmic Baryon Budget. Astrophys. J. 1998, 503, 518-530. [CrossRef]

3. Nicastro, F.; Kaastra, J.; Krongold, Y.; Borgani, S.; Branchini, E.; Cen, R.; Dadina, M.; Danforth, C.W.; Elvis, M.; Fiore, F.; et al. Observations of the missing baryons in the warm-hot intergalactic medium. Nature 2018, 558, 406-409. [CrossRef] [PubMed]

4. Ryu, D.; Schleicher, D.R.G.; Treumann, R.A.; Tsagas, C.G.; Widrow, L.M. Magnetic Fields in the Large-Scale Structure of the Universe. Space Sci. Rev. 2012, 166, 1-35. [CrossRef]

5. Widrow, L.M.; Ryu, D.; Schleicher, D.R.G.; Subramanian, K.; Tsagas, C.G.; Treumann, R.A. The First Magnetic Fields. Space Sci. Rev. 2012, 166, 37-70. [CrossRef]

6. Ichiki, K.; Takahashi, K.; Ohno, H.; Hanayama, H.; Sugiyama, N. Cosmological Magnetic Field: A Fossil of Density Perturbations in the Early Universe. Science 2006, 311, 827-829. [CrossRef] [PubMed]

7. Shiromoto, Y.; Susa, H.; Hosokawa, T. Generation of Magnetic Field on the Accretion Disk around a Proto-first-star. Astrophys. J. 2014, 782, 108. [CrossRef]

8. Xu, H.; O'Shea, B.W.; Collins, D.C.; Norman, M.L.; Li, H.; Li, S. The Biermann Battery in Cosmological MHD Simulations of Population III Star Formation. Astrophys. J. Lett. 2008, 688, L57-L60. [CrossRef]

9. Gnedin, N.Y.; Ferrara, A.; Zweibel, E.G. Generation of the Primordial Magnetic Fields during Cosmological Reionization. Astrophys. J. 2000, 539, 505-516. [CrossRef]

10. Langer, M.; Aghanim, N.; Puget, J.L. Magnetic fields from reionisation. Astron. Astrophys. 2005, 443, 367-372. [CrossRef]

11. Kulsrud, R.M.; Cen, R.; Ostriker, J.P.; Ryu, D. The Protogalactic Origin for Cosmic Magnetic Fields. Astrophys. J. 1997, 480, 481-491. [CrossRef]

12. Vazza, F.; Brunetti, G.; Brüggen, M.; Bonafede, A. Resolved magnetic dynamo action in the simulated intracluster medium. Mon. Not. R. Astron. Soc. 2018, 474, 1672-1687. [CrossRef]

13. Donnert, J.; Dolag, K.; Lesch, H.; Müller, E. Cluster magnetic fields from galactic outflows. Mon. Not. R. Astron. Soc. 2009, 392, 1008-1021. [CrossRef]

14. Miniati, F.; Bell, A.R. Resistive Magnetic Field Generation at Cosmic Dawn. Astrophys. J. 2011, $729,73$. [CrossRef]

15. Xu, H.; Li, H.; Collins, D.C.; Li, S.; Norman, M.L. Turbulence and Dynamo in Galaxy Cluster Medium: Implications on the Origin of Cluster Magnetic Fields. Astrophys. J. Lett. 2009, 698, L14-L17. [CrossRef]

16. Akahori, T.; Nakanishi, H.; Sofue, Y.; Fujita, Y.; Ichiki, K.; Ideguchi, S.; Kameya, O.; Kudoh, T.; Kudoh, Y.; Machida, M.; et al. Cosmic magnetism in centimeter- and meter-wavelength radio astronomy. Publ. Astron. Soc. Jpn. 2018, 70, R2. [CrossRef]

17. Yamazaki, D.G.; Kajino, T.; Mathews, G.J.; Ichiki, K. The search for a primordial magnetic field. Phys. Rep. 2012, 517, 141-167. [CrossRef]

18. Takami, H.; Nishimichi, T.; Yahata, K.; Sato, K. Cross-correlation between UHECR arrival distribution and large-scale structure. J. Cosmol. Astropart. Phys. 2009, 6, 031. [CrossRef]

19. Takahashi, K.; Mori, M.; Ichiki, K.; Inoue, S. Lower Bounds on Intergalactic Magnetic Fields from Simultaneously Observed GeV-TeV Light Curves of the Blazar Mrk 501. Astrophys. J. 2012, 744, L7. [CrossRef]

20. Fujita, Y.; Ohira, Y. Radio mini-halo emission from cosmic rays in galaxy clusters and heating of the cool cores. Mon. Not. R. Astron. Soc. 2013, 428, 599-608. [CrossRef]

21. Takizawa, M. N-Body + Magnetohydrodynamical Simulations of Merging Clusters of Galaxies: Characteristic Magnetic Field Structures Generated by Bulk Flow Motion. Astrophys. J. 2008, 687, 951-958. [CrossRef]

22. Akahori, T.; Kato, Y.; Nakazawa, K.; Ozawa, T.; Gu, L.; Takizawa, M.; Fujita, Y.; Nakanishi, H.; Okabe, N.; Makishima, K. ATCA $16 \mathrm{~cm}$ observation of CIZA J1358.9-4750: Implication of merger stage and constraint on non-thermal properties. Publ. Astron. Soc. Jpn. 2018, 70, 53. [CrossRef]

23. Sofue, Y.; Machida, M.; Kudoh, T. The Primordial Origin Model of Magnetic Fields in Spiral Galaxies. Publ. Astron. Soc. Jpn. 2010, 62, 1191-1201. [CrossRef] 
24. Vernstrom, T.; Gaensler, B.M.; Brown, S.; Lenc, E.; Norris, R.P. Low-frequency radio constraints on the synchrotron cosmic web. Mon. Not. R. Astron. Soc. 2017, 467, 4914-4936. [CrossRef]

25. Hong, S.E.; Kang, H.; Ryu, D. Radio and X-Ray Shocks in Clusters of Galaxies. Astrophys. J. 2015, 812, 49. [CrossRef]

26. Akahori, T.; Ryu, D.; Gaensler, B.M. Fast Radio Bursts as Probes of Magnetic Fields in the Intergalactic Medium. Astrophys. J. 2016, 824, 105. [CrossRef]

27. Ryu, D.; Kang, H.; Cho, J.; Das, S. Turbulence and Magnetic Fields in the Large-Scale Structure of the Universe. Science 2008, 320, 909-912. [CrossRef] [PubMed]

28. Haverkorn, M.; Spangler, S.R. Plasma Diagnostics of the Interstellar Medium with Radio Astronomy. Space Sci. Rev. 2013, 178, 483-511. [CrossRef]

29. Han, J.L. Observing Interstellar and Intergalactic Magnetic Fields. Annu. Rev. Astron. Astrophys. 2017, 55, 111-157. [CrossRef]

30. Gaensler, B.M.; Beck, R.; Feretti, L. The origin and evolution of cosmic magnetism. New Astron. Rev. 2004, 48, 1003-1012. [CrossRef]

31. Feretti, L.; Giovannini, G.; Govoni, F.; Murgia, M. Clusters of galaxies: Observational properties of the diffuse radio emission. Astron. Astrophys. Rev. 2012, 20, 54. [CrossRef]

32. Vacca, V.; Murgia, M.; Govoni, F.; Feretti, L.; Giovannini, G.; Orra, E.; Bonafede, A. The intracluster magnetic field power spectrum in Abell 665. Astron. Astrophys. 2010, 514, A71. [CrossRef]

33. Govoni, F.; Dolag, K.; Murgia, M.; Feretti, L.; Schindler, S.; Giovannini, G.; Boschin, W.; Vacca, V.; Bonafede, A. Rotation measures of radio sources in hot galaxy clusters. Astron. Astrophys. 2010, 522, A105. [CrossRef]

34. Beck, R. Magnetic Visions: Mapping Cosmic Magnetism with Lofar and Ska. In Proceedings of the Conference Magnetic Fields in the Universe II: From Laboratory and Stars to the Primordial Universe, Cozumel, Mexico, 28 January-1 February 2008.

35. Carilli, C.L.; Taylor, G.B. Cluster Magnetic Fields. Annu. Rev. Astron. Astrophys. 2002, 40, 319-348. [CrossRef]

36. Akahori, T.; Ryu, D. Faraday Rotation Measure due to the Intergalactic Magnetic Field. II. The Cosmological Contribution. Astrophys. J. 2011, 738, 134. [CrossRef]

37. Akahori, T.; Ryu, D. Faraday Rotation Measure Due to the Intergalactic Magnetic Field. Astrophys. J. 2011, 723, 476-481. [CrossRef]

38. Wilman, R.J.; Miller, L.; Jarvis, M.J.; Mauch, T.; Levrier, F.; Abdalla, F.B.; Rawlings, S.; Klöckner, H.-R.; Obreschkow, D.; Olteanu, D.; et al. A semi-empirical simulation of the extragalactic radio continuum sky for next generation radio telescopes. Mon. Not. R. Astron. Soc. 2008, 388, 1335-1348. [CrossRef]

39. Cho, J.; Ryu, D. Characteristic Lengths of Magnetic Field in Magnetohydrodynamic Turbulence. Astrophys. J. Lett. 2009, 705, L90-L94. [CrossRef]

40. Akahori, T.; Ryu, D.; Kim, J.; Gaensler, B.M. Simulated Faraday Rotation Measures toward High Galactic Latitudes. Astrophys. J. 2013, 767, 150. [CrossRef]

41. Vazza, F.; Brüggen, M.; Hinz, P.M.; Wittor, D.; Locatelli, N.; Cheller, C. Probing the origin of extragalactic magnetic fields with Fast Radio Bursts. Mon. Not. R. Astron. Soc. 2018, 480, 3907-3915. [CrossRef]

42. Akahori, T.; Gaensler, B.M.; Ryu, D. Statistical Techniques for Detecting the Intergalactic Magnetic Field from Large Samples of Extragalactic Faraday Rotation Data. Astrophys. J. 2014, 790, 123. [CrossRef]

43. Ravi, V.; Shannon, R.M.; Bailes, M.; Bannister, K.; Bhandari, S.; Bhat, N.D.R.; Burke-Spolaor, S.; Caleb, M.; Flynn, C.; Jameson, A.; et al. The magnetic field and turbulence of the cosmic web measured using a brilliant fast radio burst. Science 2016, 354, 1249-1252. [CrossRef] [PubMed]

44. Johnston-Hollitt, M.; Govoni, F.; Beck, R.; Dehghan, S.; Pratley, L.; Akahori, T.; Heald, G.; Agudo, I.; Bonafede, A.; Carretti, E.; et al. Using SKA Rotation Measures to Reveal the Mysteries of the Magnetised Universe. In Proceedings of the Advancing Astrophysics with the Square Kilometre Array (AASKA14), Giardini Naxos, Italy, 9-13 June 2014.

45. Taylor, A.R.; Stil, J.M.; Sunstrum, C. A Rotation Measure Image of the Sky. Astrophys. J. 2009, 702, $1230-1236$. [CrossRef]

46. Oppermann, N.; Junklewitz, H.; Greiner, M.; Enßlin, T.A.; Akahori, T.; Carretti, E.; Gaensler, B.M.; Goobar, A.; Harvey-Smith, L.; Johnston-Hollitt, M.; et al. Estimating extragalactic Faraday rotation. Astron. Astrophys. 2015, 575, A118. [CrossRef]

47. Hammond, A.M.; Robishaw, T.; Gaensler, B.M. A New Catalog of Faraday Rotation Measures and Redshifts for Extragalactic Radio Sources. arXiv 2012, arXiv:1209.1438. 
48. Beck, A.M.; Lesch, L.; Dolag, K.; Kotarba, H.; Geng, A.; Stasyszyn, F.A. Origin of strong magnetic fields in Milky Way-like galactic haloes. Mon. Not. R. Astron. Soc. 2012, 422, 2152-2163. [CrossRef]

49. Machida, M.; Akahori, T.; Nakamura, K.E.; Nakanishi, H.; Haverkorn, M. Radio broadband visualization of global three-dimensional magneto-hydrodynamical simulations of spiral galaxies II. Faraday Depolarization from $100 \mathrm{MHz}$ to $10 \mathrm{GHz}$. arXiv 2018, arXiv:1811.02829.

50. Machida, M.; Akahori, T.; Nakamura, K.E.; Nakanishi, H.; Haverkorn, M. Radio broadband visualization of global three-dimensional magnetohydrodynamical simulations of spiral galaxies-I. Faraday rotation at 8 GHz. Mon. Not. R. Astron. Soc. 2018, 480, 17-25. [CrossRef]

51. Akahori, T.; Kumazaki, K.; Takahashi, K.; Ryu, D. Exploring the intergalactic magnetic field by means of Faraday tomography. Publ. Astron. Soc. Jpn. 2014, 66, 65. [CrossRef]

52. Farnes, J.S.; O'Sullivan, S.P.; Corrigan, M.E.; Gaensler, B.M. Faraday Rotation from Magnesium II Absorbers toward Polarized Background Radio Sources. Astrophys. J. 2014, 795, 63. [CrossRef]

53. Zhu, G.; M'enard, B.M. The JHU-SDSS Metal Absorption Line Catalog: Redshift Evolution and Properties of Mg II Absorbers. Astrophys. J. 2013, 770, 130. [CrossRef]

54. Schnitzeler, D.H.F.M. The latitude dependence of the rotation measures of NVSS sources. Mon. Not. R. Astron. Soc. 2010, 409, L99-L103. [CrossRef]

55. Mao, S.A.; Gaensler, B.M.; Haverkorn, M.; Zweibel, E.G.; Madsen, G.J.; McClure-Griffiths, N.M.; Shukurov, A.; Kronberg, P.P. A Survey of Extragalactic Faraday Rotation at High Galactic Latitude: The Vertical Magnetic Field of the Milky Way Toward the Galactic Poles. Astrophys. J. 2010, 714, 1170-1186. [CrossRef]

56. Stil, J.M.; Taylor, A.R.; Sunstrum, C. Structure in the Rotation Measure Sky. Astrophys. J. 2011, 726, 4. [CrossRef]

57. Sotomayor-Beltran, C.; Sobey, C.; Hessels, J.W.T.; de Bruyn, G.; Noutsos, A.; Alexov, A.; Anderson, J.; Asgekar, A.; Avruch, I.M.; Beck, R.; et al. Calibrating high-precision Faraday rotation measurements for LOFAR and the next generation of low-frequency radio telescopes. Astron. Astrophys. 2013, 552, A58. [CrossRef]

58. Brentjens, M.A.; de Bruyn, A.G. Faraday rotation measure synthesis. Astron. Astrophys. 2005, 441, 1217-1228. [CrossRef]

59. Burn, B.J. On the depolarization of discrete radio sources by Faraday dispersion. Mon. Not. R. Astron. Soc. 1966, 133, 67-83. [CrossRef]

60. Miyashita, Y.; Ideguchi, S.; Takahashi, K. Performance test of RM CLEAN and its evaluation with chi-square value. Publ. Astron. Soc. Jpn. 2016, 68, 44. [CrossRef]

61. Sun, X.H.; Rudnick, L.; Akahori, T.; Anderson, C.S.; Bell, M.R.; Bray, J.D.; Farnes, J.S.; Ideguchi, S.; Kumazaki, K.; O'Brien, T.; et al. Comparison of Algorithms for Determination of Rotation Measure and Faraday Structure. I. 1100-1400 MHz. Astron. J. 2015, 149, 60. [CrossRef]

62. Kumazaki, K.; Akahori, T.; Ideguchi, S.; Kurayama, T.; Takahashi, K. Properties of intrinsic polarization angle ambiguities in Faraday tomography. Publ. Astron. Soc. Jpn. 2014, 66, 61. [CrossRef]

63. Fransworth, D.; Rudnick, L.; Brown, S. Integrated Polarization of Sources at $\lambda \sim 1 \mathrm{~m}$ and New Rotation Measure Ambiguities. Astron. J. 2011, 141, 191. [CrossRef]

64. Heald, G.; Braun, R.; Edmonds, R. The Westerbork SINGS survey. II Polarization, Faraday rotation, and magnetic fields. Astron. Astrophys. 2009, 503, 409-435. [CrossRef]

65. Akahori, T.; Ideguchi, S.; Aoki, T.; Takefuji, K.; Ujihara, H.; Takahashi, K. Optimum Frequency of Faraday Tomography to Explore the Inter-Galactic Magnetic Field in Filaments of Galaxies. arXiv 2018, arXiv:1808.10546.

66. Ideguchi, S.; Takahashi, K.; Akahori, T.; Kumazaki, K.; Ryu, D. Fisher analysis on wide-band polarimetry for probing the intergalactic magnetic field. Publ. Astron. Soc. Jpn. 2014, 66, 5. [CrossRef]

(C) 2018 by the author. Licensee MDPI, Basel, Switzerland. This article is an open access article distributed under the terms and conditions of the Creative Commons Attribution (CC BY) license (http:/ / creativecommons.org/licenses/by/4.0/). 